\title{
La inteligencia empresarial en las pequeñas y medianas empresas competitivas de América Latina - algunas reflexiones ${ }^{*}$
}

\section{Julio Cubillo}

\section{Resumo}

Neste artigo, desenvolvem-se algumas teses preliminares que, na opinião do autor, podem ser úteis a muitos profissionais da região da América Latina e do Caribe, envolvidos nos trabalhos de geração, processamento, comercialização e administração dos conhecimentos e das informações necessárias às pequenas e médias empresas. Considera-se também que algumas das idéias aqui propostas interessem aos empresários que poderiam beneficiar-se de uma reflexão individual e conjunta com os "trabalhadores do conhecimento e da informação" sobre estes temas na região.

\section{Palavras-chave}

Inteligência empresarial; Inteligência competitiva; Pequenas e médias empresas; América Latina e Caribe; Informação, Conhecimento.

\section{Contexto}

1. La pequeña y mediana empresa (PYME) es considerada hoy día como uno de los actores clave del desarrollo. (Nota 1). Esta percepción se sustenta tanto en el examen de indicadores económico-sociales (incidencia en el empleo, en el PIB y en el comercio exterior, ...) como en su consolidación como tema relevante en las agendas del desarrollo. (Nota 2)

2. El nuevo contexto internacional en que se mueven los negocios plantean grandes desafíos a las PYMES de América Latina. El futuro de éstas se definirá cuando hayan puesto a prueba su capacidad de competir en economías crecientemente desreguladas y abiertas a los intercambios con el exterior. La lucha se dará tanto en el mercado interno (competencia de empresarios extranjeros) y en los mercados internacionales (liderazgo de procesos importadores y exportadores de bienes y servicios por parte de la PYME).

3. El enfrentamiento de formas nuevas de competencia con actores externos cada vez más capaces ponen a prueba en grado crítico a las PYMES para po-

* Artigo apresentado no seminário internacional "Gestão Estratégica do Conhecimento", patrocinado pelo Senai/Ciet - Centro Internacional para a Educação, Trabalho e Transferência de Tecnologia, e pelo MCT/Finep - Financiadora de Estudos e Projetos, em 03 e 04/07/97, Rio de Janeiro/RJ.

Los puntos de vista expresados en este artículo son estrictamente personales y no reflejan necesariamente los puntos de vista de la organización en que trabaja el autor. El texto no ha sido revisado por los servicios editoriales de la Cepal. sicionarse y articularse con otros actores de su entorno, tales como proveedores, clientes y competidores. (Porter). Ello exige a la PYMES el dominio y el ejercicio de una capacidad continua de monitoreo o vigilancia del entorno. Se trata de percibir, interpretar y transformar en decisiones a las señales fuertes y débiles indicativas de variaciones en el entorno, ya sea provengan del llamado microambiente (espacio sobre el cual la PYME puede ejercer algún grado de control ) o del macroentorno (espacio que es un dato para la PYME). (Sainte-Marie)

4. El tema del manejo del conocimiento y la información como ingrediente central de una gestión empresarial en las PYMES latinoamericanas debe ser percibido como una salida al escaso margen de maniobra que estas organizaciones tienen para sobrevivir en un mundo globalizado. Según un estudio reciente el poco margen de maniobra de las PYMES reside en "la capacidad de la gerencia de desarrollar estrategias competitivas" y en la "capacidad de articular acuerdos, básicamente entre agentes del sector privado, para construir cadenas en que cada eslabón incremente el valor de la producción" (Rosales). La ingeniería de estrategias y acuerdos pasa netamente por una fortalecida capacidad de inteligencia empresarial en las PYMES.

Ha motivado la preparación de este trabajo un conjunto de percepciones, (todavía incompletas y poco conexas), de que este tema no ha sido abordado ni con la intensidad ni con la profundidad que amerita al menos en la región que nos preocupa: la América Latina y el Caribe. 
Nuestra impresión es que, salvo excepciones, los empresarios de las PYMES latinoamericanas se enfrentan muy desprotegidos y en un estado de alta vulnerabilidad al interactuar en un cambiante mercado del conocimiento y de la información, y con pocas herramientas para sacar provecho de las informaciones y conocimientos externos. La complejidad y mutabilidad de los entornos de las PYMES hacen cada vez más imperioso para él que toma decisiones contar allí con "nuevas cartas e instrumental de navegación".

El dilema es que, frente a esta necesidad imperiosa, el empresario de las empresas medianas y pequeñas, por lo general:

1) no está consciente de la importancia del tema de la inteligencia empresarial, y de todas las facetas que este incluye, (aunque ciertamente se da cuenta de aspectos parciales como la importancia de contar a tiempo con información que le permita hacer sus compras correctamente, estar actualizado con la mejor tecnología de producción y sistemas administrativos modernos, conocer los precios para sus productos y los sustitutos, etc.).

2) no dispone de los recursos para crear en su empresa una unidad especializada permanente en el tema de inteligencia empresarial.

3) no dispone de las "tecnologías ni de la cultura de uso de conocimientos e información", ni tampoco maneja, quizás por lo mismo, una información completa y fidedigna sobre el mercado de proveedores de conocimientos e información, lo que conlleva a que su comportamiento como "consumidor" de conocimientos e informaciones provistas por actores externos no sea coherente ni informado.

En este artículo se desarrollan algunas tesis preliminares que se estima pueden ser útiles para muchos profesionales de la región involucrados en los trabajos de generación, procesamiento, comercialización, y gestión de los conocimientos e informaciones necesarios para las PYMES y que estén interesados en un acercamiento a tales empresas ya sea para asesorarlas, trabajar en ellas o preparar los recursos humanos que allí se necesitan.
También se piensa que algunas de las ideas aquí propuestas puedan ser de interés para los dueños-empresarios, esto es los dirigentes de las PYMES quienes se podrían beneficiar de una reflexión individual y conjunta con los "trabajadores del conocimiento y de la información" sobre estos temas en la región latinoamericana y caribeña.

\section{INTELIGENCIA EMPRESARIAL}

\subsection{Concepto de Inteligencia empresarial}

El monitoreo continuo de las señales del entorno - sobre todo de aquellas que permitan anticipar una situación futura, ya sea para reaccionar o actuar propositivamente frente al medio - es ejercido por un conjunto de capacidades que la empresa debe poner en marcha, y que entenderemos como inteligencia empresarial (Nota 3).

La inteligencia empresarial, designa, en nuestro entender, aquel conjunto de capacidades propias o movilizables por una entidad lucrativa, destinadas a asegurar el acceso, capturar, interpretar y preparar conocimiento e información con alto valor agregado para apoyar la toma de decisiones requeridas por el diseño y ejecución de su estrategia competitiva (Nota 4).

\subsection{Percepciones alternativas sobre el trabajo de inteligencia empresarial}

Todavía subyacen, visiones alternativas poco integrables acerca de los trabajos de inteligencia empresarial

Según cierta vertiente de pensamiento (la de la transferencia de información) la inteligencia consistiría en captar (por medios lícitos) (Nota5) el conocimiento y la información obtenible en mercados públicos (bienes públicos y bienes transables por un precio) donde sería posible encontrar cerca del $90 \%$ de los insumos de información y conocimiento que la empresa necesita. Una vez "'empaquetada" esta información se le transferiría, esto es, se le haría llegar físicamente al decisor quien podría así "consumirla" sin más antes de tomar una decisión acertada.
Se piensa, entonces que el trabajo de inteligencia consistiría en asegurar el acceso a sistemas de acceso al conocimiento y la información, formales, públicos y privados: la contratación de expertos, la compra de servicios de acceso a bases de datos, consulta de bibliotecas, etc.

Otra vertiente es la aportada por las comunicaciones sociales, preferentemente por aquella rama que se preocupa de las "relaciones públicas". Allí la empresa es percibida como un actor político capaz no sólo de captar señales sino, además, de crear y transmitir hacia el entorno una imagen deseada de la organización. En esta visión se trata, en definitiva, de la búsqueda por parte de la organización de un posicionamiento en la mente del consumidor y de la sociedad en general, de un conjunto de signos que diferencien nítidamente a la empresa de otros entes afines o asociados. Los comunicados corporativos, la publicidad, los informes anuales de la gerencia al directorio, las conferencias de prensa conforman un "paquete comunicacional" que es enviado en forma direccionada hacia espacios simbólicos donde también están presentes productos comunicacionales de otras empresas.

La corriente de "transferencia de la información" concibe al trabajo de inteligencia como una interacción informativa con el medio gobernada por la captación o "succión" de la información del entorno para procesarla, y transferirla al tomador de decisión. En la corriente "comunicacional" el centro de gravedad del trabajo de interacción informativa de la empresa con el entorno está puesto en la captación de mensajes de otras corporaciones y la emisión de mensajes desde la empresa hacia el entorno conforme una política de "comunicaciones" al servicio del "proyecto político" de los dirigentes de la empresa.

Estas dos posiciones no reflejan desde luego realidades tan separables. Por ejemplo en la "escuela de la transferencia de la información" hay muchos elementos comunicacionales. Toda captura de información, por mas sigilosamente que sea hecha, es, en sí misma, un acto comunicacional. Quien captura información por cualquier medio de que se trate "está siendo obser- 
vado" por otros actores cualesquiera que sea su rol (proveedor, cliente, competidor, regulador estatal). También hay elementos comunicacionales en la interacción de esta rama del trabajo de inteligencia con los actores internos de la organización. No sólo deberá poder capturar información y conocimiento interno (ya sea que verse sobre el entorno o sobre el ambiente interno de la empresa), sino que deberá poder establecer canales abiertos y expeditos de comunicación con los actores formuladores de la estrategia competitiva y los dirigentes empresariales. Deberá saber hacer el "empaquetamiento del conocimiento y la información", colocando dicho producto informativo de alto valor agregado en el contexto de un "discurso político", breve, motivador, sugerente y expresado en el lenguaje del decisor.

En las escuelas de "las relaciones públicas" también existe la necesidad de ejercer procesos de observación del entorno empresarial, monitorear los símbolos que están siendo generados por otros actores (campañas de propaganda, discursos de directorio, comunicados, home pages de Internet) de los clientes proveedores, competidores, reguladores estatales. Hay también, por lo tanto, captura de información, procesamiento y transmisión de mensajes a instancias internas de la empresa. Probablemente este trabajo se apoyará menos en la consulta a proveedores y sistemas formales de información y más a la observación participante, la investigación acción, la detección del rumor, un contacto permanente con otros informadores, a menudo establecido mediante relaciones informales nojerárquicas".

\section{INTELIGENCIA EMPRESARIAL Y GRAN EMPRESA}

\subsection{Las cabezas-múltiples de la inteligencia empresarial en la gran empresa}

En una empresa grande, los roles representados por ambas escuelas de pensamiento están frecuentemente diferenciados y son ejercidos desde unidades separadas y administrativamente desconectadas.
La unidad (interna o externa) que opere conforme la escuela de la "transferencia de información" le proporcionará al tomador de decisión observaciones sobre actos del cliente registrados en sistemas formales: indicadores de sus tres últimos balances; publicaciones que generó el personal de la empresa cliente; patentes que subscribió; sociedades que constituyó, etc.

La unidad de la escuela "relaciones públicas" le suministrará, por otra parte, información de prensa sobre el cliente; discursos pronunciados por su gerencia; viajes realizados por funcionarios de la empresa; rumores que se dicen sobre tal empresa; gastos en publicidad de dicha empresa; etc.").

La alta gerencia de la gran empresa o la empresa transnacional deberá usualmente tratar de integrar dos o más informes complementarios de inteligencia sobre la situación de, por ejemplo, una empresa cliente. El dirigente empresarial, además, estará en posición de cubrir no sólo los gastos de producción internos de informes de inteligencia complementarios. También podrá hacerse asesorar por un especialista de alto nivel para tener una perspectiva más cabal de la situación y curso de acción de tal empresa cliente. Asimismo la gran empresa gozará de un acceso a un activo humano (redes de negocios) y estará en condiciones de operar importantes acervos de tecnologías de información y telecomunicaciones (Gran potencia de cómputos y redes telemáticas) (Blanc).

En el caso que la gran empresa decida subcontratar servicios externos de inteligencia empresarial también encontrará una cierta segmentación en el mercado del conocimiento e información relevantes. Frecuentemente también estos mercados reflejan la división histórica de las disciplinas de la información existiendo, por ejemplo: brokers de información, analistas financieros (escuela de transferencia de información); analistas de prensa, analistas políticos (escuela de relaciones públicas). Hay desde luego segmentos del mercado de conocimientos y de información donde se perfilan situaciones híbridas en los productos y servicios ofrecidos (analistas de mercado, asesores de gerencia).

\subsection{Un modelo integrado de unidad de inteligencia empresarial en la gran empresa}

La gran empresa se caracteriza por una especialización de las funciones asociadas a la formulación de la estrategia competitiva y a los trabajos de inteligencia empresarial.

En este trabajo se asumirá una visión abarcadora del área de inteligencia empresarial. Esta corresponde a un grupo interdisciplinario integrado por profesionales de la escuela "transferencia del conocimiento y la información" (especialistas substantivos generadores de conocimiento, bibliotecólogos, estadísticos, informáticos) y por profesionales de la escuela de comunicaciones-relaciones públicas que provengan de las ciencias mercadológicas y de las comunicaciones sociales.

Este equipo humano será capaz de generar un "único" informe de inteligencia integrado cubriendo todas las facetas necesarias para el decisor y la unidad de formulación de la estrategia empresarial. Tal informe será estructurado conforme un discurso político adecuado al estilo cognoscitivo del decisor o del experto de la unidad de formulación de la estrategia empresarial. Idealmente deben posibilitar establecer asociaciones entre sus partes y conceptos y la exploración de contenidos con diferente grado de profundidad (hipertextos). El paradigma actual de un informe de inteligencia de esta naturaleza sería un archivo accesible en red de computadoras a través de un esquema Intranet o Internet con acceso regulado.

Para entender mejor las relaciones entre los actores que contextualizan el trabajo de inteligencia en una gran empresa resulta útil elaborar un esquema o modelo simplificado de la situación. Es ésta sin duda una visión idealizada que puede no ser necesariamente válida para todas las grandes empresas. 
Una gran empresa se puede así representar o modelar en una forma desagregada destacando los núcleos de oferta y de demanda de servicios y productos de inteligencia empresarial. Tales capacidades se ilustran en la figura 1.

Los flujos de conocimientos e información (informes, consejos de viva voz, adiestramiento, etc..) son los que se indican en la página siguiente:

Flujo I i-E: Captura directa de información del entorno por unidad de inteligencia interna

Flujo I I-PI: Captura indirecta de información vía proveedor de servicios de información de valor agregado bajo o medio

Flujo I x-E: Captura de información del entorno por proveedor de servicios de inteligencia externo

Flujo I i-Ix: Servicios de inteligencia suministrados por proveedor externo

Flujo I i- F: servicios internos integrados de inteligencia ofrecidos al área de formulación de estrategia competitiva

Flujo I I-D: servicios internos integrados de inteligencia ofrecidos al área de toma de decisiones

Flujo F-D: interacciones para la formulación y la ejecución de la estrategia competitiva

En el contexto de la gran empresa se puede suponer que existen condiciones para:

I. subcontratar servicios externos de inteligencia

II. subcontratar servicios externos de información de valor agregado bajo o medio

III. adquirir substanciales conocimientos e informaciones directamente del entorno interno o de la organización propiamente tal

IV. atender en forma flexible a las dos clientelas internas principales: el área de toma de decisiones y el área de formulación de estrategias competitivas.

FIGURA 1

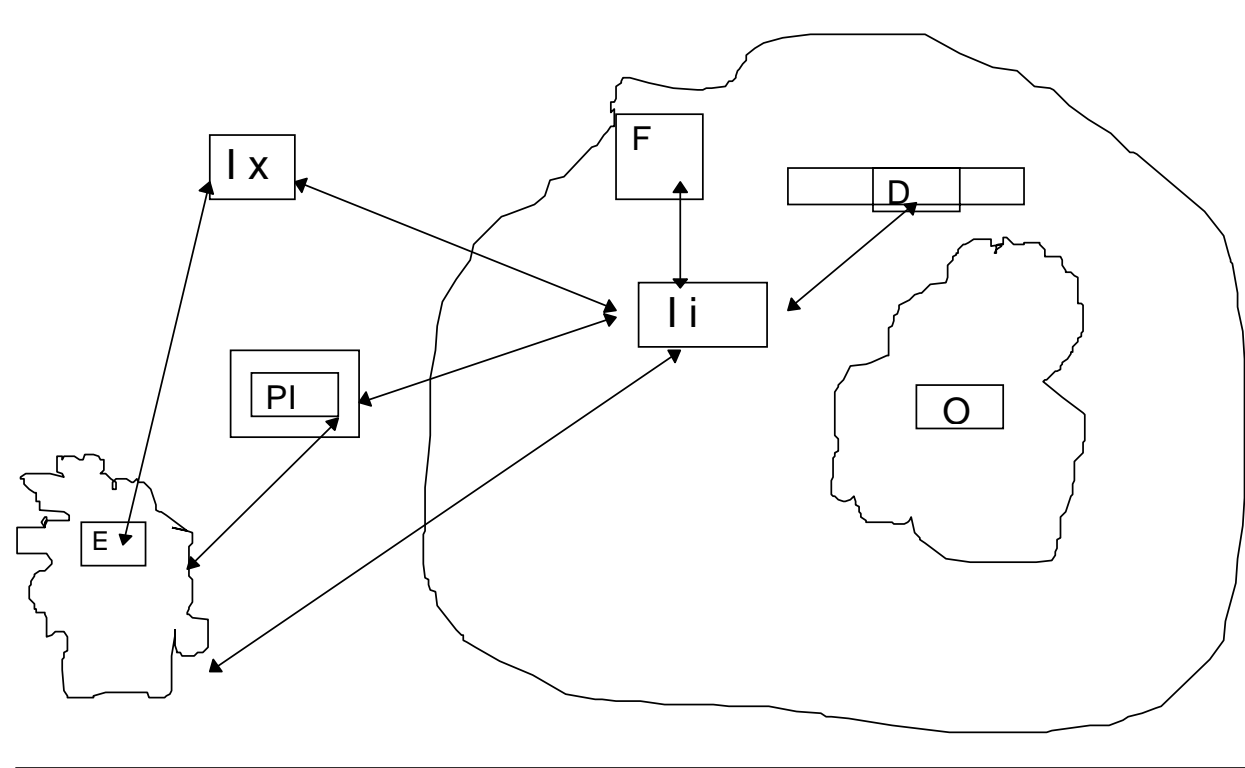

D: Area de toma de decisiones

F: Area de formulación de la estrategia competitiva (unidad de planeamiento estratégico, por ejemplo,..)

I I: Area de Inteligencia integrada (ejerce su acción cognoscitiva endógenamente sobre la organización $\mathrm{O}$ y endógenamente sobre el entorno $\mathrm{E}$

P I: Proveedor de servicios de información sobre el entorno de valor agregado bajo o medio I x: Proveedor externo de Información de inteligencia subcontratado por la unidad I i

La gran empresa podría albergar unidades internas de inteligencia especializada por área funcional (marketing, producción, desarrollo tecnológico,..) y por area geográfica (unidades ubicadas en gerencias de subsedes o filiales de la gran empresa)

Además, se puede suponer allí la existencia de un sistema de unidades de inteligencia que definen sus propios métodos para conocer el entorno externo e interno, para subcontratar con proveedores externos de servicios de inteligencia y para atender las necesidades de las clientelas principales (área de toma de decisiones y área de formulación de la estrategia competitiva).

El funcionamiento eficaz del núcleo I i dependerá de factores tales como:

I. Sólido conocimiento del mercado de proveedores de servicios de inteligencia empresarial

II. Sólidos conocimientos del mercado de proveedores de servicios de información

III. Sólidos conocimientos de las técnicas de monitoreo directo del entorno externo e interno
IV. Conocimiento y comprensión de la estrategia competitiva

V. Manejo de los procesos comunicacionales para manejar las relaciones con clientela interna, proveedores y competidores internos (unidades de inteligencia afines)

En este mundo de la gran empresa surgen claros nichos de mercado para diferentes profesionales del conocimiento y de la información que deseen asumir los roles requeridos por estas unidades de inteligencia empresarial internas. (Cronin, 1988).

\section{INTELIGENCIA EMPRESARIAL EN EL MUNDO DE LAS PYMES}

\subsection{Las PYMES y sus circunstan- cias: modelo simple de inteligencia empresarial}

En una PYME se tendrá probablemente la situación que se presenta en la figura siguiente:

Allí se observa que:

I. la toma de decisiones, la formulación de la estrategia empresarial y el trabajo de inteligencia empresarial serán 
asumidos prácticamente en forma íntegra por el dueño-empresario.

II. la inteligencia empresarial del entorno y del medio interno será llevada a cabo directamente por el dueño empresario (observación, emisión de mensaje, búsqueda de información por canales informales y formales)

III. son débiles las vinculaciones con actores externos del mercado de conocimientos e información (empresas de inteligencia, proveedores convencionales de productos y servicios de información).

Esta situación presenta ventajas y desventajas. Entre las ventajas destacan que hay poca intermediación y que es el dueño empresario el gran sintetista del complejo estrategia competiva-inteligencia y toma de decisiones. Entre las desventajas están que el dueño empresario confía en sus capacidades directas de observación del medio, principalmente a través de mecanismos informales de visitas a clientes, conversación con proveedores, reuniones con otros empresarios de su clase y usa poco o nada la información existente en sistemas formales o el conocimiento provisto por asesores. Ello plantea limitaciones, debido al escaso tiempo que podrá destinar a observación y a sistematizar información. Por otro lado, la complejidad del entorno requeriría acceso a acervos informativos más amplios, particularmente cuando hablamos de mercados internacionales.

3.2 Los papeles múltiples del dueñoempresario en el trabajo de inteligencia y posibles nichos de mercado para "trabajadores del conocimiento y de la información"

Nos imaginamos que el dueño empresario deberá estar preparado para desempeñar alguna de las siguientes funciones:

I. Orquestrador de la inteligencia empresarial.

En ese rol deberá tener una visión de un "mapa" de conocimientos e informaciones sobre el entorno y el medio ambiente interno en función de la estrategia competitiva empresarial que tenga en mente. Ello implica planificar los re-

\section{FIGURA 2}

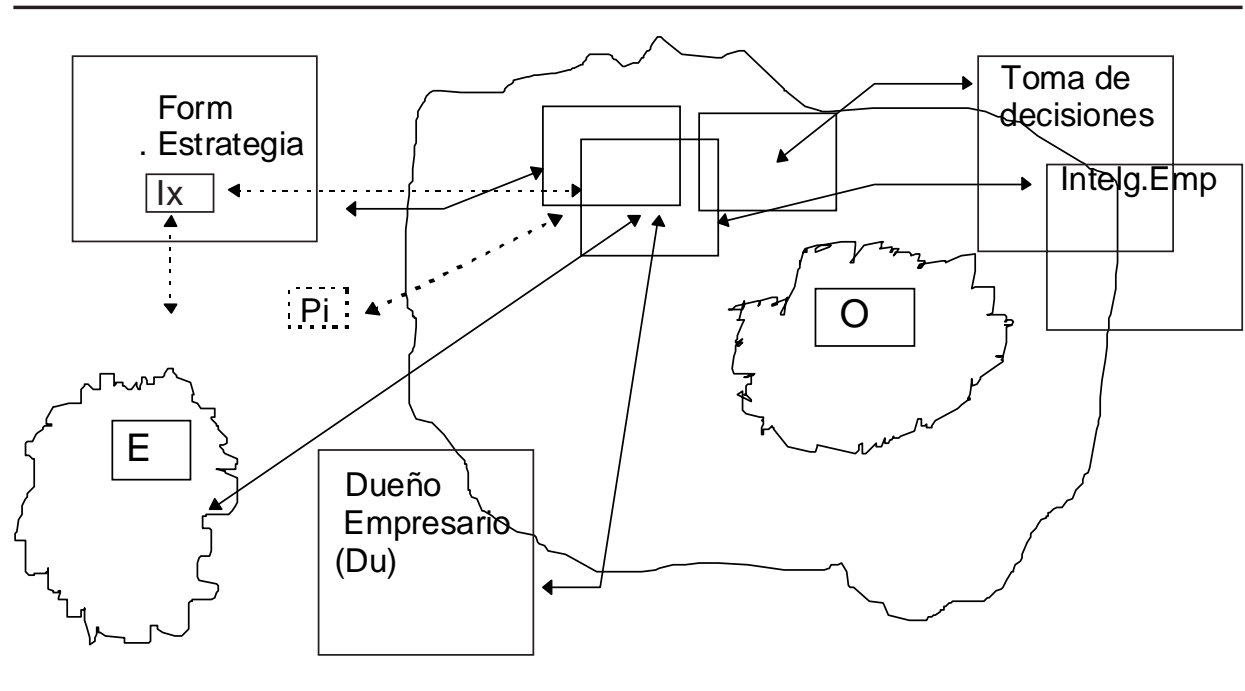

sultados esperados de inteligencia, a quién designará para la ejecución de los mismos y qué recursos se asignarán para tales fines.

Aquí existe la necesidad de concebir y poner a prueba métodos prácticos, rápidos y de bajo costo mediante los cuales los empresarios puedan construir los mapas de inteligencia en su empresa (interesante nicho para la investigación-acción y la capacitación). También es de interés trabajar con los propios empresarios algunos criterios con los cuales se puede determinar el valor potencial del conocimiento y de la información en situaciones específicas de uso del conocimiento y la información. Definitivamente en éste y otros ámbitos de la inteligencia empresarial las propuestas eventuales de los trabajadores del conocimiento y la información tendrán mayor posibilidad de éxito si son diseñadas CON los empresarios y no aisladamente por los profesionales técnicos, por sí y ante sí mismos, PARA los empresarios.

II. Subcontratista de servicios de inteligencia empresarial y de proveedores externos de productos y servicios de información de valor bajo o medio

Este rol supone del empresario estar familiarizado con los mercados de conocimientos y de informaciones de interés para su empresa. Ello implica conocer no sólo los eventuales proveedores, sino que además saber algo sobre la calidad de los productos y servicios ofrecidos, los precios de acceso y los costos de utilización de los mismos en la empresa. Naturalmente que la adquisición de productos informativos de menos valor agregado supondrán un mayor procesamiento interno en la empresa y por lo tanto un mayor costo de utilización de los mismos.

Hemos detectado (vía observación asistemática en el terreno) que existe poco conocimiento en los empresarios de las PYMES latinoamericanas sobre la oferta de conocimientos e información por parte de proveedores externos, y un escaso convencimiento de que es rentable el gasto en estos servicios. Hay poca cultura de uso de mecanismos formales de suministros de conocimientos e información. Se sigue confiando en las conversaciones de almuerzos y eventos, más que en los productos de información sistemáticamente elaborados. Naturalmente esta situación varía de país en país, existiendo casos donde el gobierno,las universidades o los propios gremios empresariales han hecho esfuerzos para facilitar el acceso a la información a los empresarios.

También el empresario ha de estar consciente de que este conocimiento sobre los mercados del conocimiento y de la información se facilita y se facilitará más aún en el futuro mediante el uso del Internet y las redes electrónicas internacionales en general. Sin embargo, habrá que pensar en subsidios o ayudas para que el empresario de las PYMES, usualmente presionado por el tiempo y la escasez de recursos, saque provecho de los beneficios mas seguros 
y evidentes del Internet. El mercadeo irresponsable del Internet como LA respuesta para el acceso al conocimiento y la información en el mundo empresarial puede sumir a los líderes de las PYMES en una gran confusión en un mar caótico y casi ilimitado de información.

De utilidad, además, será que los empresarios desarrollen capacidades negociadoras para establecer contratos de largo plazo con brokers, consultores y proveedores de información a fin de asegurar continuidad, calidad de servicios y precios razonables.

En este rubro, pensamos, habrían interesantes posibilidades para oferentes potenciales de capacitación en la tipificación de servicios de acceso al conocimiento y la información, el uso del INTERNET y la negociación de acuerdos con proveedores.

\section{iii. Operador del trabajo de inteligencia empresarial}

El empresario de una PYME latinoamericana que busca mantenerse competitiva en los mercados internos e internacionales deberá ser capaz de asumir una buena parte del trabajo de inteligencia. Ha de estar consciente de aquello que ha venido haciendo en materia de inteligencia empresarial a fin de descubrir sus áreas de fortaleza y debilidad. Una suerte de 'auditoría' del trabajo de inteligencia desempeñado por el empresario podría ser de gran utilidad.

En esta área de inteligencia empresarial directamente operada por el empresario de PYME es donde surge con fuerza el desarrollo de una concepción integradora del trabajo de inteligencia que reúna y transcienda el aporte de los enfoques de "transferencia de información" y de comunicaciones-relaciones públicas". Al ser un "outsider" en el mundo de los conocimientos y la información (sólo remotamente ocurrirá que su procedencia por formación y experiencia estuviese adscrito a una de las escuelas de pensamiento-acción existentes sobre esta materia), el dueño empresario estará en óptimas condiciones de adoptar un enfoque integrado y desprejuiciado del trabajo de inteligencia empresarial.
En este terreno hay "nichos de mercado" para actividades de apoyo al empresariado. Habría que fortalecerlo en las técnicas de investigación- acción, enseñarle el arte del discurso político y capacitarlo a utilizar las fuentes de información formal e informal.

iv. Estimulador de una "equipo" de inteligencia basado en el personal de su empresa

La situación de ser operador del trabajo de inteligencia coloca al dueño-empresario en una posición de alta exigencia que se suma a las múltiples actividades que debe cumplir en su empresa. Será así vital para él que logre comprender mejor la naturaleza del trabajo de inteligencia empresarial a fin de quedar en posición de delegar algunas de sus actividades en sus empleados (modelo japonés participativo de las funciones de observación). Esta asignación de tareas de inteligencia habrá de hacerse en todos aquellos rubros donde la subcontratación de servicios externos no sea posible o cuando ello no se justifique en términos técnicos y económicos.

En particular, estamos pensando en el frente de observación del entorno de la empresa donde todos los empleados de una PYME, independientemente de su función, podrían ejercer capacidades de observación de eventos. Conforme reglas éticas claras los empleados pueden ser motivados para realizar observaciones con relación a clientes, proveedores, empresas competidoras, medios de comunicación pública formales e institutos de investigación y desarrollo potenciales generadores de productos o suministros alternativos.

En esta área el empresario deberá ser un estimulador y un docente para instruir a sus empleados a llevar a cabo estos trabajos. Existe aquí la necesidad potencial de preparar esquemas de entrenamiento para que los empresarios puedan a su vez entrenar a sus empleados, o pautas a ser comunicadas directamente a los empleados por un agente externo contratado por el empresario para tales efectos.

v. Consumidor de los productos de inteligencia-empresarial
El empresario se beneficiará, por último, si sabe especificar un producto o servicio de inteligencia empresarial, y sabe utilizar los contenidos recibidos en el momento del suministro.

Se piensa que podría ser útil llevar a cabo seminarios talleres con empresarios donde se les exponga a diferentes productos de inteligencia y se les enseñe a armar interpretaciones de los datos, y las ideas. Cabe destacar, que se distingue entre un producto de información de valor agregado medio o bajo (como el que es accesible a través de fuentes públicas, rentadas o no, tales como bibliotecas, "home pages' del INTERNET, y bases de datos de diferente tipo y complejidad). El producto de inteligencia empresarial se caracteriza por valores agregados de contextualización, asociación e interpretación que van mucho más allá de los productos informativos convencionales. Es la construcción de una nueva percepción no evidente del entorno a través de la combinación de conocimientos y productos informativos dispersos "hasta construir un nuevo mosaico" (Pontigo)

En este terreno se buscaría aproximarse a desarrollar las capacidades que autores consideran habría de poseer el "sintetista" y "reconocedor de patrones". En este tema ya se han formulado visiones que han ido probando tener validez en la medida que avanzamos en el tiempo. Por ejemplo se ha dicho: "Nunca más (el oficial de inteligencia técnicoeconómica) deberá preocuparse de diseñar caminos y medios para asegurar el acceso a la información, construir canales privilegiados y proteger el secreto de sus fuentes. El estará preocupado por el procesamiento de vastas cantidades de datos, en verificar y comparar diferentes fuentes a fin de elegir la más confiable y la menos onerosa, y en establecer interconexiones entre una variedad de asuntos y eventos de particular relevancia.."(Sagasti)

\section{REFLEXIONES FINALES}

En esta etapa preliminar de nuestro análisis se podrían extraer algunas hipótesis que se podría intentar constrastar en futuros esfuerzos de investigación que a lo mejor resulta de interés llevar a cabo por parte de académicos o estudiantes de programas de postgrado 
en las disciplinas vinculadas con la información.

a) Un monitoreo del entorno ligado sólidamente a la estrategia competitiva de la empresa, es un ingrediente esencial para la supervivencia y desarrollo de las PYMES en los medios aceleradamente mutantes en que se vive y se vivirá en la primera década del 2000.

b) El trabajo de inteligencia empresarial es radicalmente distinto en la $P Y M E$ con relación a la gran empresa, no solamente por los recursos que están al alcance de esta última sino que por las diferencias notables en los microambientes, macroambientes y ámbito de la estrategia competitiva.

c) Un funcionamiento más fluido y transparente de los mercados del conocimiento y la información será esencial para los trabajos de inteligencia de las PYMES. Un mercado más accesible y con más opciones favorecerá que se generen y transen allí los productos y servicios adecuados a las estrategias competitivas de las PYMES.

d) El mercado de conocimientos e información funcionará mejor en la medida que:

- todo dueño-empresario de una PYME tenga al menos un conocimiento del territorio en que se debe llevar a cabo el trabajo de inteligencia que realize o subcontrate su empresa, y debe poder moverse en los mercados del conocimiento que son relevantes a su organización.

- los proveedores de conocimientos e información y de servicios de acceso al conocimiento y la información para las PYMES deben a su vez conocer los ámbitos de las estrategias competitivas de las PYMES y dónde el conocimiento y la información del entorno es un factor crítico

- se generen opciones para el acceso al conocimiento y la información a través de consorcios de PYMES que pueden en conjunto conseguir acuerdos con los proveedores de conocimientos y de productos y servicios de información. e) Existen numerosos nichos potenciales para que los trabajadores del conocimiento y de la información puedan brindar apoyo a las PYMES. Desde luego ya no será suficiente asegurar el acceso a las fuentes de información. La batalla por los mercados se dará en los segmentos de mayor complejidad y mayor valor agregado de productos y servicios.

\subsection{Epílogo}

Hemos acometido este trabajo después de algún tiempo de observación (asistemática) del accionar de las PYMES. En la medida que lo hemos desarrollado nos han ido surgiendo muchas interrogantes e inquietudes para el futuro.

En este primer abordaje al tema de la inteligencia aplicado al mundo de las PYMES nos hemos preocupado preferentemente de las relaciones entre dos actores: los dueños empresarios y los trabajadores del conocimiento y de la información. Hemos dejado deliberadamente fuera, por ser un tema que ameritaría un espacio mucho mayor del disponible, el examinar el papel del Estado en este tema. Tampoco hemos examinado el espacio que podría caberle a la cooperación inter-empresarial que se visualiza como de enorme trascendencia en éste y otros campos. (Hanna).

En el plano netamente conceptual nos asaltan serias dudas sobre la validez de la clasificación de las PYMES según el criterio de tamaño para fines de tipificar y organizar el trabajo de inteligencia empresarial. En el tema del uso del conocimiento y la información intervienen otras variables que determinarán los "mapas de inteligencia" en las pequeñas y medianas empresas. Sólo podríamos mencionar en esta etapa a dos que se nos ocurren: la dependencia de las PYMES a cadenas productivas de grandes empresas: la intensidad de uso de conocimientos e información que requieran las diferentes funciones básicas de la empresa tales como mercadeo, manufactura, finanzas, etc. Todas éstas son áreas de pesquisa aplicada podrían incentivarse en las universidades latinoamericanas.
Nos queda, por último, una percepción final. Es necesario un despertar de los dos actores examinados en este artículo hacia el tema de la inteligencia. El pequeño empresario debe tomar conciencia de lo vital del tema y de sus responsabilidades en el mismo. El trabajador del conocimiento y la información debe cambiar sus paradigmas mentales y sentirse formando parte de una comunidad compleja de proveedores de servicios y productos generados en consultas y diálogo permanente con los empresarios. La inteligencia empresarial no es simplemente hacer nuevas bases de datos con tecnologías más sofisticadas. No es tampoco solamente crear páginas web o escribir nuevos softwares. Ya se nos está agotando la estrategia de seguir haciendo más de lo mismo.

Mas bien la gestación de lo nuevo pasará por entender mejor el trabajo empresarial en las PYMES y redefinir los espacios de colaboración de las diferentes profesiones.

Pensamos que ninguna profesión vinculada a la gestión del conocimiento y la información está excluida por decreto de este juego. Sin embargo, sobrevivirán aquellas que puedan cambiar sus paradigmas, movilizar mejor conocimientos substantivos sobre áreas específicas y ejercer un mejor dominio de las tecnologías de información y comunicación.

La manifestación más temprana e intensa de nichos emergentes de productos y servicios de información y conocimientos relevantes en el mundo de las PYMES dependerá de un despertar de un sector empresario, que no ha podido todavía articular plenamente nuevas respuestas y se siente, por lo general, en situación poco confortable frente al imperativo de competir en mercados globales. Un despertar equivalente en su grado de dramatismo y urgencia se espera de los profesionales del conocimiento y la información, una nueva mirada quizás menos ingenua y más comprometida con el desafío global. 


\section{NOTAS}

\section{NOTA 4}

\section{NOTA 1}

Según Eiroles, la tipología de empresas basada en el tamaño de las mismas que ha tenido una mayor difusión es la que se apoya en e indicador: número de empleados que alberga la empresa

Micro empresa:

Pequeña empresa

Mediana empresa

Gran empresa

En este trabajo cuando hablemos de PYMES estaremos hablando de empresas hasta 25 empleados. (esto es la microempresa y las empresas menores del estrato "pequeña empresa").

\section{NOTA 2}

El impacto económico de las PYMES se puede expresar a través de diferentes indicadores. Por ejemplo, el sector PYMES es el responsable del 68,60 y $69 \%$ de los empleos remunerados de España, Italia y México. Representó por el contrario un $40 \%, 30 \%$ y $3 \%$ de las exportaciones totales de los respectivos países. Ello apuntaría a una menor productividad y capacidad exportadora de las PYMES latinoamericanas (Eiroles). Por otra parte, existe una considerable efervescencia en los medios financieros internacionales para buscar medios de fortalecer e sector PYMES nacional (ej: reuniones internacionales previstas por el BID y el Banco Mundial para los próximos meses)

\section{NOTA 3}

La inteligencia empresarial es un concepto afín a los de inteligencia organizacional y de inteligencia técnico-económica planteado en un trabajo antecesor del presente (Cubillo)

\section{Latin America small and medium size competitive enterprises and business intelligence - some thoughts}

\author{
Abstract \\ The article deals with some questions \\ involving the generation, processing, \\ marketing and management of knowledge and \\ information, oriented to Latin America and the \\ Caribbean small and medium size enterprises, \\ that can be useful for both information \\ professionals and entrepreneurs.
}

\section{Keywords}

Business Inteligence; Competitive Intelligence; Small and Medium Size

Enterprises; Latin America and the

Caribbean; Information; Knowledge.
La estrategia competitiva según un estudio reciente comprendería: "una definición de la misión de la empresa, el menú de objetivos jerarquizados de la estrategia, la estrategia de mercadeo, la estrategia de manufactura, y los criterios de competitividad de los mercados meta (precio, calidad, flexibilidad, tiempo de entrega y confiabilidad) (Rosales, pág 103-104)

\section{NOTA 5}

En este documento adoptamos la postura de que el trabajo de inteligencia empresarial debe regirse por códigos de ética compartidos por los actores del mundo de la empresa. No abogamos por una posición angélica de la inteligencia, pero tampoco inscribimos el tema en el mundo del espionaje, el sabotaje de sistemas de información u otros mecanismos nada lícitos de adquirir información o de impedir que el "adversario" pueda hacer su propio trabajo con el conocimiento y la información

\section{REFERENCIAS BIBLIOGRÁFICAS}

1. BLANC, Gérard. The grain traders: masters of the intelligence game. En Dedijer, S; Jéquier $\mathrm{N}$ (Eds). Intelligence and economic development. An inquiry into the role of the knowledge industry, Berg, Oxford, 1987. pp 139-157.

2. CRONIN, Blaise. New horizons for the information profession: strategic intelligence and competitive advantage. En: Dyer.G; Tseng, G; New horizons for the information profession. Taylor Graham, 1988, pp 3-22.

3. CUBILLO, Julio. Techno-economic Intelligence INTELL. What is in it for developing countries? Journal of economic and social intelligence, vol2, no2, 1992, pp 123-148.

4. DEDIJER, S; Jéquier, Nicholas (Eds). Intelligence and economic development. An inquiry into the role of the knowledge industry, Berg, Oxford, 1987.

5. EROLES, Antonio. La pequeña empresa exportadora. Panorama, México DF 1995.

6. HANNA, Nagy; Dagonjic, Vlasta. Why a national strategy for exploiting information technologies. Atas Bulletin, issue 10 autumn 1995, pp 32-47.

7. PONTIGO, Jaime. Información para la industria; estrategia de mercado. Ciencias de la Información, la Habana, vol 22, No 2 , junio 1991, pp 14-23.

8. PORTER, Michael; Millar, Victor. Cambiando la naturaleza de la competencia (resumen de Cepal Clades). Harvard Business Review, june 1995 pp 149-160.

9. ROSALES, Ramón. Estrategias Gerenciales para la pequeña y mediana empresa. Ediciones IESA, Caracas, 1996.

10. SAGASTI, Francisco. Techno-economic intelligence for development. En Dedijer, $\mathrm{S}$; Jéquier $\mathrm{N}$ (Eds). Intelligence and economic development. An inquiry into the role of the knowledge industry, Berg, Oxford, 1987. pp 173-183.

11. SAINTE-MARIE, Georges. Dirigir una Pyme. 10 etapas. Paidós empresa 31, Barcelona, 1995.

12. Latin America Small and Medium Size Competitive Enterprises and Business Intelligence - Some Thoughts.

\section{AGRADECIMIENTOS}

El autor agradece los comentarios de la Dra. Vania Araujo del CIET (Rio de Janeiro) y de sus colegas Luis Alba y Nelson Aguirre del Clades de la Cepal. Naturalmente los errores, omisiones o inadecuada interpretación de las ideas generosamente aportadas por estos colegas son de la exclusiva responsabilidad del autor.

Julio Cubillo 